\title{
Giant Endometrial Polyp in a Postmenopausal Woman
}

\author{
Vivek Nair ${ }^{1}$, Jitendra S. Nigam ${ }^{2}$, Jyotsna N. Bharti ${ }^{3}$, Biswajit Dey ${ }^{4}$, Ashok Singh ${ }^{5}$ \\ 1. Pathology, Vikash Multispeciality Hospital, Sambalpur, IND 2. Pathology/Lab Medicine, All India Institute of \\ Medical Sciences, Patna, IND 3. Pathology, All India Institute of Medical Sciences, Jodhpur, IND 4. Pathology, North \\ Eastern Indira Gandhi Regional Institute of Health and Medical Sciences, Shillong, IND 5. Pathology, All India Institute \\ of Medical Sciences, Rishikesh, IND
}

Corresponding author: Vivek Nair, drviveknair2001@gmail.com

\begin{abstract}
Endometrial polyps are the benign localized overgrowth of endometrial tissue composed of a variable amount of gland, fibroblast-like spindle cells stroma, and thick-walled blood vessels. They develop as a result of unbalanced estrogens and progestin. Polyps greater than $4 \mathrm{~cm}$ are considered giant polyps. We report a case of giant endometrial polyp in a postmenopausal woman who presented with postmenopausal bleeding without any history of hormone or drug intake. However, the possible cause may be the age and use of phytoestrogens in the daily routine diet for a long time.
\end{abstract}

Categories: Obstetrics/Gynecology, Pathology

Keywords: phytoestrogens, estrogens, progesterone, aging

\section{Introduction}

Endometrial polyps (EPs) are the benign localized overgrowth of endometrial tissue protruding into the uterine cavity, affecting approximately $25 \%$ of women [1,2]. EP comprises a variable amount of gland, fibroblast-like spindle cells stroma, thick-walled blood vessels, and are lined by pseudostratified active or flat inactive epithelium $[1,2]$. The pathogenesis of EP is not well known; however, they are believed to develop due to unbalanced estrogens and progestin [1,2]. Phytoestrogens (PEs), a mimic of estrogen, are produced by plants and found abundantly in spices, herbs, and food [1]. Long-term consumption of PEs can act like estrogen, causing an unbalance between estrogen and progestin, leading to uterine pathologies such as endometrial hyperplasia or EP [1]. The use of tamoxifen and raloxifene is associated with an increased frequency of giant EPs [1]. EPs are usually less than $2 \mathrm{~cm}$, and polyps greater than $4 \mathrm{~cm}$ are considered giant polyps [3]. We report the case of a giant EP in a postmenopausal woman who presented with postmenopausal bleeding without any history of hormone or drug intake.

Review began 01/10/2021 Review ended 01/18/2021 Published 01/19/2021

\section{๑) Copyright 2021}

Nair et al. This is an open access article distributed under the terms of the Creative Commons Attribution License CC-BY 4.0., which permits unrestricted use, distribution, and reproduction in any medium, provided the original author and source are credited.

\section{Case Presentation}

A 67-year-old female, P4L4, presented in the gynecology outpatient department with a history of bleeding per vaginum for one month which was associated with spasmodic abdominal pain not relieved by medication. No history of prolonged illness, drug, or hormonal intake was elicited. The patient was of normal built for age with a body mass index of 20.1. Gynecological examination was unremarkable. Routine hematological and biochemical parameters were within the normal limits. On ultrasonography (USG), a homogenous hyperechoic mass measuring $48.4 \times 38.2 \mathrm{~mm}$ with a central anechoic area attached to the fundus occupying the uterine cavity was detected. A diagnosis of submucosal fibroid was made on USG. Total abdominal hysterectomy was done, and the specimen was sent for histopathological examination. We received the total hysterectomy specimen. The uterus with cervix measured $11 \times 7 \times 5 \mathrm{~cm}$ with $1.5 \mathrm{~cm}$ long cervical canal. On cutting, a pedunculated polypoidal mass with a thin stalk attached to the fundus area, measuring $7 \times 4 \times 4 \mathrm{~cm}$, was seen in the endometrial cavity. The external surface of the mass was smooth. The cut surface was grayish white with multiple small cystic spaces filled with altered blood.

Microscopically, the cervix showed features of chronic non-specific cervicitis. The endometrium showed simple endometrial hyperplasia for age. The polyp showed variable-sized glands lined by cuboidal-tocolumnar cells with variable spindle cells stroma and several blood vessels (Figure 1). 


\section{Cureus}
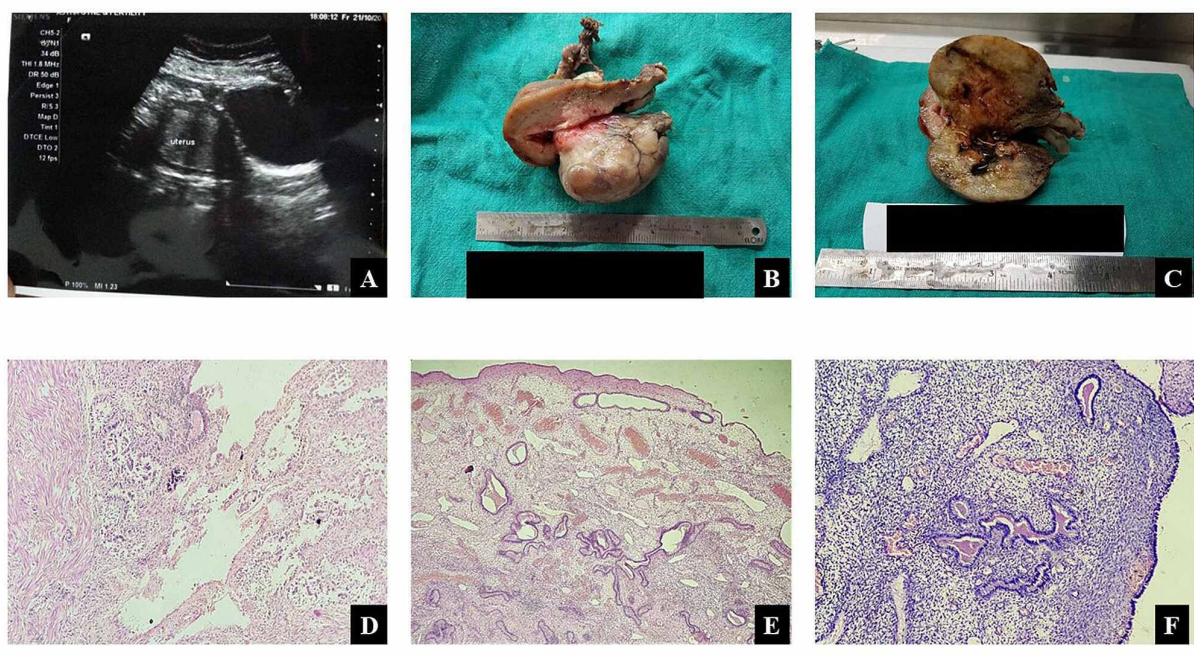

\section{FIGURE 1: (A) The homogenous hyperechoic mass with a central anechoic area attached to the fundus occupying the uterine cavity. (B) A pedunculated polypoidal mass with a thin stalk attached to the fundus area. (C) Multiple small cystic spaces filled with altered blood. (D) Myometrium with endometrium and stalk of the EP. (E \& F) Variable- sized glands with variable spindle cells stroma and many blood vessels. \\ $\mathrm{EP}$, endometrial polyp}

No atypia was noted in all the sections examined. A histopathological diagnosis of simple endometrial hyperplasia for age with benign hyperplastic EP was made. Further history was elicited from the patient for the possible etiology. She gave a history of daily intake of turmeric, garlic, ginger, onion, and the occasional intake of thyme and soybean in her diet, which are rich in PEs. Based on the clinical and histopathological findings, a final diagnosis of benign hyperplastic EP probably caused by the long-term dietary intake of PEs was made. The postoperative period was uneventful and the patient was doing well after six months of follow-up.

\section{Discussion}

EPs are a common benign endometrial pathology that affects approximately $25 \%$ of women $[2,4]$. They are generally asymptomatic but can be seen with abnormal uterine bleeding in $13-50 \%$ of the cases. EPs may also present with infertility, premalignant, and malignant endometrial lesions [2]. In the present case, the patient presented with abnormal uterine bleeding associated with spasmodic abdominal pain without any history of prolonged illness, drug, or hormonal intake. The pathogenesis of EPs is not well known. However, by a systematic, semi-quantitative review on the pathogenesis of EP, Indraccolo et al. concluded that in postmenopausal women or during the first phase of the menstrual cycle, hyperactivation of $\beta$ estrogen receptors on the $\alpha$ receptor enhance estrogen sensitivity in some areas of the endometrium leading to polyp formation [5]. Apoptosis via BCL-2 gene expression is blocked by estrogen-related inflammation, preventing their shedding during menstruation [5]. Indraccolo et al. also observed that there is a causative link between EP and BCL-2 expression, obesity, unbalanced estrogen therapy, imbalance between estrogen and progestins, estrogen-like effect, the relationship between estrogen and progestins, and tamoxifen, regardless of the timing [5]. Giant EPs are rare and are mostly associated with tamoxifen and raloxifene treatment [1]. Aging has also been linked to EPs [5]. In the present case, the patient was a 67-year-old postmenopausal female, and aging may be linked to the giant EP formation in the patient. The normal body mass index of the patient and normal biochemical results ruled out the possibility of any metabolic disorder. Certain plant-derived phytochemicals known as PEs are functionally and structurally similar to $17 \beta$ eestradiol (isoflavones) or synthetic estrogens such as diethylstilboestrol (lignins) [4]. Grains, fiber-rich foods, legumes, and nuts used in the diet rich in PEs present as glycosides [4]. Thyme, turmeric, garlic, ginger, onion, soy, pomegranate, licorice, red clover, hops, and Verbena are rich sources of PEs and phytoprogestins, which act as agonists and antagonists in vivo [6]. These are commonly consumed foods, herbs, and spices in an average Indian diet. Their activity in a female depends on their concentration, endogenous estrogen concentration, and menopausal status [1]. PEs with their estrogen-like effect can cause an imbalance with progesterone, which may lead to endometrial hyperplasia in postmenopausal women [1]. In the present case, there was a history of daily intake of turmeric, garlic, ginger, onion, and the occasional intake of thyme and soybean in her diet, which may be the possible cause of EP. Aging, obesity, arterial hypertension, postmenopausal period, and tamoxifen are also risk factors for malignancy development in 
the EP [2]. However, malignancy in EPs is uncommon and seen in 1-3\% of the cases [2]. EPs larger than 15 $\mathrm{mm}$ are associated with endometrial hyperplasia, and in general, polyps measuring more than $10 \mathrm{~mm}$ are associated with an increased incidence of malignancy [2]. In the present case, no atypia or malignancy was seen after extensive sampling.

\section{Conclusions}

The origin and pathogenesis of EPs are still not fully understood. However, in our case, we assume that aging and use of PEs in the daily routine diet for a long time may be the cause of the giant EP.

\section{Additional Information \\ Disclosures}

Human subjects: Consent was obtained or waived by all participants in this study. Conflicts of interest: In compliance with the ICMJE uniform disclosure form, all authors declare the following: Payment/services info: All authors have declared that no financial support was received from any organization for the submitted work. Financial relationships: All authors have declared that they have no financial relationships at present or within the previous three years with any organizations that might have an interest in the submitted work. Other relationships: All authors have declared that there are no other relationships or activities that could appear to have influenced the submitted work.

\section{References}

1. Unal B, Dogan S, Karaveli FS, et al.: Giant endometrial polyp in a postmenopausal woman without hormone/drug use and vaginal bleeding. Case Rep Obstet Gynecol. 2014, 2014:518398. 10.1155/2014/518398

2. Lasmar BP, Lasmar RB: Endometrial polyp size and polyp hyperplasia . Int J Gynaecol Obstet. 2013, 123:236239. 10.1016/j.ijgo.2013.06.027

3. Cil AS, Bozkurt M, Kara D, Guler B: Giant endometrial polyp protruding from the external cervical os in a postmenopausal woman: magnetic resonance imaging and hysteroscopic findings. Proc Obstet Gynecol. 2013, 3:1-9. 10.17077/2154-4751.1230

4. Burton JL, Wells M: The effect of phytoestrogens on the female genital tract . J Clin Pathol. 2002, 55:401407. 10.1136/jcp.55.6.401

5. Indraccolo U, Di Iorio R, Matteo M, Corona G, Greco P, Indraccolo SR: The pathogenesis of endometrial polyps: a systematic semi-quantitative review. Eur J Gynaecol Oncol. 2013, 34:5-22.

6. Zava DT, Dollbaum CM, Blen M: Estrogen and progestin bioactivity of foods, herbs, and spices . Proc Soc Exp Biol Med. 1998, 217:369-378. 10.3181/00379727-217-44247 